\title{
Methods and madness: agitation, delirium, and postoperative cognitive dysfunction
}

\author{
Gregory L. Bryson, MD
}

Published online: 5 June 2010

(C) Canadian Anesthesiologists' Society 2010

"If you can't describe what you are doing as a process, you don't know what you're doing." A

W. Edwards Deming

"Though this be madness, yet there is method in "t."

William Shakespeare, Hamlet, Act 2, scene 2

Over the past decade, disorders of cognition in adult surgical patients have attracted the attention of perioperative physicians. As our surgical population ages and lengths of hospital stay decrease, postoperative cognitive deficits present a barrier to efficient discharge and return to function in the community. Physicians are aware that treatment of delirium adds $\$ 2,500$ US to hospital stays of patients $>65 \mathrm{yr}$ of age, costing Medicare approximately $\$ 6.9$ billion US annually. ${ }^{1}$ Similarly, we know that patients with postoperative cognitive dysfunction (POCD) one week following surgery are more likely to leave employment, while those with POCD persisting three months postoperatively suffer an increased risk of mortality in the subsequent five years. ${ }^{2}$ In this month's issue of the Journal, $\mathrm{Yu}$ et al. present an observational cohort study of 2,000 patients undergoing general anesthesia. Their study results indicate that $>20 \%$ of adult subjects experienced postanesthetic agitation. ${ }^{3}$

Delirium, POCD, agitation-the variety of cognitive disorders studied in the perioperative period can be...well...rather confusing. Often the terms are defined poorly and sometimes they are used interchangeably. Is agitation in the postanesthesia care unit synonymous with delirium? Is emergence agitation, like delirium, associated with significant adverse outcomes? While emergence

G. L. Bryson, MD ( $\square)$

Department of Anesthesiology, The Ottawa Hospital - Civic

Campus, 1053 Carling Avenue, Box 249C, Ottawa,

ON K1Y 4E9, Canada

e-mail: glbryson@ottawahospital.on.ca

agitation may not be representative of normal cognition, is it POCD? What is the busy clinician to make of all this? Perhaps a good beginning would be to takee the advice of management expert, W. Edwards Deming, and describe the process under discussion.

Only one of the conditions mentioned in the preceding paragraph is a diagnosable psychiatric disorder. The Diagnostic and Statistical Manual of Mental Disorders defines delirium as a transient, fluctuating disturbance of consciousness, attention, cognition, and perception. ${ }^{4}$ The Confusion Assessment Method (CAM) ${ }^{5}$ operationalizes the diagnosis of delirium using four clinical features: 1) acute onset and fluctuating course; 2) inattention; 3) disorganized thinking; and 4) altered level of consciousness. Diagnosis is based on the presence of features 1 and 2 plus one of either 3 or 4 . The CAM has been used extensively, and its sensitivity and specificity both exceed $90 \% .^{5}$ On the other hand, POCD is a more subtle condition and more difficult to quantify.

While inherently challenging, POCD research has been hampered further by the large number of cognitive tests employed and the widely disparate definitions of an abnormal test result. An operating definition of POCD has emerged based on a decade of research by the International Study of Postoperative Cognitive Dysfunction (ISPOCD). ${ }^{6}$ The ISPOCD research group employs a battery of four individual neuropsychometric tests given before and after surgery and standardizes interval changes in test scores using data from a non-surgical control group. While the deficits in attention, concentration, executive function, verbal memory, visuospatial abstraction, and psychomotor speed are both real and quantifiable, POCD as a disorder is

\footnotetext{
A “W. Edwards Deming." Quotes.net. STANDS4 LLC, 2010. Available from URL: http://www.quotes.net/quote/8537 (accessed April 2010).
} 
largely a research construct. What then is emergence agitation?

Inappropriate motor behaviour, disorientation, and emotional lability are frequently associated with emergence from anesthesia in children. Yu et al. expand their inquiry to the adult population and define emergence agitation as "...combative behaviour, thrashing, and hyperactive motor behaviour". They then graded agitation according to the following scheme: a) mild: in response to stimulation; b) moderate: occurring without stimulation and lasting for at least five minutes but not requiring intervention; and, c) severe: lasting for at least five minutes and requiring drugs and/or physical restraint. ${ }^{3}$ Using this definition, $\mathrm{Yu}$ found that 212 of 2,000 adult subjects $(10.6 \%)$ experienced mild agitation, $178(8.9 \%)$ experienced moderate agitation, and $36(1.8 \%)$ experienced severe agitation. The frequency of agitation noted in the present study was fourfold greater than previous research in adults. ${ }^{7}$ These results would suggest an underreported and clinically significant problem similar in scope to delirium or POCD. What should the clinician make of these results?

First, as this discussion has progressed from delirium to emergence agitation, there has been a transition from a psychiatric disorder to a single behaviour. For a diagnosis of delirium to be considered, agitation, representing an abnormal level of consciousness, must be combined with an acute and fluctuating course and inattention. It is unclear in the present study if these other criteria were assessed or present, so it is inappropriate to equate the present findings with the body of research evaluating delirium.

Second, the scale used to assess the behaviour of interest in the present study was not designed to be used in adults. ${ }^{8}$ In fact, the three-point scale was modified from a trial recruiting children two to nine years of age where agitation was described as " $1=$ calm, $2=$ agitated but consolable, and 3 = severely agitated and inconsolable". Duration and therapy was not implied in the original citation, and it is unclear what one would do to console a combative adult. Choice of this tool as a primary outcome measure in an adult population is difficult to defend. As the authors themselves indicate, there are a number of other validated scores for the assessment of agitation in adults. ${ }^{9,10}$ While these other tools are used predominantly in critical care settings, the use of a tool designed and validated for the assessment of agitation in adults would make it easier to equate the present findings to other adult populations.

Third, pediatric researchers have advocated strongly for use of a standardized tool for the assessment of emergence agitation. Two recent systematic reviews ${ }^{11,12}$ identifying over fifty randomized controlled trials and over 5,300 patients highlight the variability in both assessment tools and definition of outcome. Kuratani and Oi identify a significant limitation to the findings, i.e., “...most studies used a self-made non validated rating scale to evaluate emergence agitation, which focused more on behavioural than on psychometric factors". ${ }^{11}$ The authors of both studies call for the use of the Pediatric Anesthesia Emergence Delirium (PAED) ${ }^{13}$ scale in future research. The PAED scale, developed in 2004 by researchers from the Hospital for Sick Children, Toronto, evaluates five behaviours (eye contact, purposeful action, awareness of surroundings, restlessness, and consolability) that are scored from 1 to 4 . Increasing PAED scores are correlated with increased agitation. The PAED scale was designed not only to assess agitation but also to address the diagnostic criteria for delirium. The PAED score correlates with bedside observers' estimates of the severity of emergence reaction and post-discharge behaviour disturbances. Interrater reliability of the PAED was excellent (i.e., 0.84, 95\% confidence intervals [CI], 0.76-0.90), and a score of 10 or more was $64 \%$ sensitive and $86 \%$ specific for postanesthesia care unit nurses to use sedative medications. The use of the PAED in the present study would have broadened the scope of the primary outcome and would have made the results more directly comparable with the body of pediatric research on this topic.

Lastly, the investigators provided no data regarding supplemental sedative use, nursing workload, or adverse patient outcomes related to emergence agitation. Including these clinical outcomes would have helped to place $\mathrm{Yu}$ et al.'s findings into context. In their absence, it is difficult to determine if emergence delirium is a significant clinical problem in adult anesthesia or if it is simply an annoying and short-lived epiphenomenon of general anesthesia.

Despite these limitations, Yu's findings raise some interesting questions. What is the mechanism of emergence agitation? Does the rate of emergence from anesthesia matter? More rapid emergence from anesthesia with a low solubility inhalational agent such as sevoflurane is associated with an increased risk of emergence agitation in children (odds ratio [OR] 2.21, 95\% CI 1.77-2.77), ${ }^{11}$ yet co-administration of midazolam seems to have no effect (OR 0.88, 95\% CI 0.44-1.76). ${ }^{12}$ What components of analgesia are essential in preventing emergence agitation? A recent meta-analysis suggests that centrally active analgesics, such as opioids, ketamine, and alpha 2 adrenergic agonists, decrease emergence agitation. ${ }^{12}$ Is a regional anesthesia technique that provides analgesia without central nervous system depression also protective? Does agitation in the postanesthesia care unit predict delirium later in the postoperative period or predict POCD in the longer term?

To grapple with these questions, clinical researchers must employ more rigorous methods and instruments to ensure that measurements have internal validity. Use of instruments, such as the CAM, PAED, and critical care 
agitation scores, will generate outcomes that are clearly defined and comparable with other research in the field. Expanding the scope of inquiry beyond the phenomenon itself to include resource utilization, adverse events, and correlation with other postoperative cognitive events will help to place emergence agitation in the appropriate clinical context. Once the clinical problem has been described clearly and its natural history defined, work on the mechanisms of emergence agitation can proceed. With apologies to Shakespeare, perioperative cognitive research will advance only if there is method in its madness.

\section{Méthode et folie: l'agitation, le delirium et le dysfonctionnement cognitif postopératoire}

«Si vous ne pouvez décrire ce que vous faites comme un processus, vous ne savez pas ce que vous faites. ${ }^{\mathrm{A}}$

W. Edwards Deming

«Bien que ce soit de la folie, on y voit tout de même de la méthode. »

William Shakespeare, "Hamlet », acte II, scène 2

Au cours de la dernière décennie, les troubles cognitifs chez les patients adultes après chirurgie ont attiré l'attention des spécialistes en médecine périopératoire. Alors que notre population chirurgicale vieillit et que les durées d'hospitalisation raccourcissent, les troubles cognitifs postopératoires constituent un obstacle à un congé et un retour efficaces au fonctionnement au sein de la communauté. Les médecins sont conscients que le traitement du delirium ajoute 2500 \$ US aux séjours en hôpital des patients $>65$ ans, ce qui coûte environ 6,9 milliards de dollars US par an à Medicare. ${ }^{1}$ De même, nous savons que les patients souffrant de dysfonctionnement cognitif postopératoire (DCPO) une semaine après la chirurgie présentent un risque plus élevé de quitter leur emploi, alors que ceux souffrant d'un DCPO persistant trois mois après l'opération présentent un risque plus élevé de mortalité au cours des cinq années suivantes. ${ }^{2}$ Dans ce numéro du Journal, Yu et coll. présentent une étude de cohorte observationnelle portant

sur 2000 patients subissant une anesthésie générale. Les résultats de leur étude indiquent que $>20 \%$ des patients adultes ont manifesté une agitation après l'anesthésie. ${ }^{3}$

Le delirium, le DCPO, l'agitation-la diversité des troubles cognitifs étudiés en période périopératoire peut

\footnotetext{
A “W. Edwards Deming." Quotes.net. STANDS4 LLC, 2010. Available from URL: http://www.quotes.net/quote/8537 (accessed April 2010)
}

porter...disons...à confusion. Trop souvent, les termes sont mal définis; parfois, ils sont confondus l'un pour l'autre. L'agitation en salle de réveil est-elle synonyme de delirium? L'agitation au réveil est-elle, comme le delirium, associée à un pronostic significativement défavorable? Bien que l'agitation au réveil ne soit pas le signe d'une cognition normale, est-elle pour autant un DCPO? Que doit faire le clinicien occupé de toutes ces choses? Une bonne façon de s'atteler au problème pourrait être de suivre le conseil de W. Edwards Deming, l'expert en gestion, et de décrire le processus en question.

Une seule des affections mentionnées dans le paragraphe précédent est un trouble psychiatrique qui peut être diagnostiqué. Le Manuel diagnostique et statistique des troubles mentaux (DSM-IV) définit le delirium comme une perturbation transitoire et fluctuante de la conscience, de l'attention, de la cognition et de la perception. ${ }^{4}$ La Méthode d'évaluation de la confusion $(\mathrm{CAM})^{5}$ définit de façon mesurable le diagnostic du delirium à l'aide de quatre éléments cliniques: 1) début aigu et déroulement fluctuant; 2) inattention; 3) pensée désorganisée; et 4) niveau de conscience altéré. Le diagnostic se fonde sur la présence des éléments 1 et 2 plus 3 ou 4 . La méthode CAM a beaucoup été utilisée et sa sensibilité et sa spécificité sont toutes deux supérieures à $90 \% .^{5}$ Le DCPO, en revanche, est une affection plus subtile et plus difficile à quantifier.

Bien qu'ardue par nature, la recherche sur le DCPO a été encore ralentie par le nombre impressionnant de tests cognitifs employés et les définitions très hétérogènes de ce qui constitue un résultat de test anormal. Une définition fonctionnelle du DCPO a vu le jour à la suite d'une décennie de recherches menées par l'ISPOCD (International Study of Postoperative Cognitive Dysfunction). ${ }^{6}$ Le groupe de recherche de l'ISPOCD se sert d'un arsenal composé de quatre tests neuropsychométriques individuels réalisés avant et après la chirurgie et normalise les changements d'intervalle dans les résultats de tests à partir de données provenant d'un groupe témoin non chirurgical. Alors que les troubles de l'attention, de la concentration, de la fonction exécutive, de la mémoire verbale, de l'abstraction visuo-spatiale et la vitesse psychomotrice sont à la fois réels et quantifiables, le DCPO est, en tant que trouble, principalement un concept de recherche. Qu'entend-on donc par agitation au réveil?

Un comportement moteur inadapté, la désorientation et la labilité émotionnelle sont souvent associés au réveil après une anesthésie chez l'enfant. Yu et coll. vont plus loin et incluent la population adulte dans leur recherche. Ils définissent l'agitation au réveil comme «... un comportement belliqueux, un affolement et un comportement moteur hyperactif ». Ils ont ensuite évalué le degré d'agitation selon l'échelle suivante: a) légère: en réaction à une stimulation; b) modérée: survenant sans stimulation 
et durant au moins cinq minutes mais ne nécessitant pas d'intervention; et c) grave: durant au moins cinq minutes et nécessitant des médicaments ou un moyen de contention. ${ }^{3}$ En se fondant sur cette définition, Yu a découvert que 212 des 2000 patients adultes $(10,6 \%)$ ont fait preuve d'une agitation légère, $178(8,9 \%)$ d'une agitation modérée, et $36(1,8 \%)$ d'une agitation grave. La fréquence des épisodes d'agitation notée dans cette étude était quatre fois plus élevée que dans les recherches précédentes menées auprès de populations adultes. ${ }^{7}$ Ces résultats semblent suggérer que l'agitation constitue un problème insuffisamment rapporté mais aussi significatif d'un point de vue clinique que le delirium ou le DCPO. Que doit faire le clinicien de ces résultats?

Tout d'abord, cette discussion s'étant éloignée du delirium pour s'arrêter à l'agitation au réveil, il y a eu transition d'un trouble psychiatrique à un comportement unique. Pour qu'un diagnostic de delirium soit envisagé, il faut que l'agitation, représentant un niveau de conscience anormal, soit combinée à une évolution et une inattention aiguës et fluctuantes. L'étude de Yu et coll. ne mentionne pas si ces critères ont été évalués ou étaient présents; dès lors, il ne convient pas de comparer ces résultats au corpus de recherche portant sur le delirium.

Deuxièmement, l'échelle utilisée dans cette étude pour évaluer le comportement en question n'a pas été conçue pour une utilisation chez l'adulte. ${ }^{8}$ En fait, l'échelle en trois points a été modifiée à partir d'une étude portant sur des enfants de deux à neuf ans, dans laquelle l'agitation était décrite comme suit: $« 1=$ calme, $2=$ agité mais consolable, et $3=$ gravement agité et inconsolable ». La durée et le traitement ne faisaient pas partie de l'étude originelle, et nous ne savons pas ce qu'une personne pourrait faire pour consoler un adulte belliqueux. Le choix de cet outil en tant que mesure des résultats principaux dans une population adulte est donc difficile à défendre. Comme les auteurs le mentionnent, il existe de nombreux autres scores validés pour évaluer l'agitation chez l'adulte. ${ }^{9,10}$ Quoique ces autres outils soient principalement utilisés dans des contextes de soins critiques, l'utilisation d'un outil conçu et validé pour l'évaluation de l'agitation chez l'adulte faciliterait la comparaison entre les résultats de l'étude en question ici et d'autres populations d'adultes.

Troisièmement, les chercheurs en pédiatrie défendent ardemment l'utilisation d'un outil standardisé pour évaluer l'agitation au réveil. Deux revues méthodiques récentes ${ }^{11,12}$ ont identifié plus de cinquante études randomisées contrôlées et plus de 5300 patients, soulignant la variabilité aussi bien du point de vue des outils d'évaluation que de la définition du devenir. Kuratani et Oi notent une restriction considérable à ces résultats, soit le fait que « ... pour l'évaluation de l'agitation au réveil, la plupart des études ont utilisé une échelle maison non validée, qui se concentrait davantage sur des facteurs comportementaux que psychométriques ».11 Les auteurs de ces deux études préconisent l'utilisation de l'échelle PAED (Pediatric Anesthesia Emergence Delirium) ${ }^{13}$ dans les études futures. L'échelle PAED, mise au point en 2004 par des chercheurs de l'Hôpital des enfants malades (Hospital for Sick Children) de Toronto, évalue cinq comportements (contact visuel, action déterminée, conscience de l'environnement, impatience et capacité à être consolé), notés de 1 à 4. Des scores PAED plus élevés sont corrélés à une agitation accrue. L'échelle PAED a été conçue non seulement pour évaluer l'agitation, mais également pour permettre d'identifier des critères diagnostiques pour le delirium. Le score PAED est corrélé aux estimations des observateurs au chevet quant à la gravité de la réaction au réveil et aux troubles comportementaux après le congé de l'hôpital. La fiabilité inter-observateur de l'échelle était excellente (soit 0,84, intervalle de confiance [IC] $95 \%, 0,76-0,90$ ), et un score de 10 ou plus était sensible à $64 \%$ et spécifique à $86 \%$, ce qui a permis aux infirmières de la salle de réveil de savoir s'il fallait utiliser des agents sédatifs. L'utilisation de l'échelle PAED dans l'étude de Yu et coll. aurait permis d'accroître la portée du critère d'évaluation principal, tout comme elle aurait permis une comparaison plus directe de leurs résultats au corpus de recherche pédiatrique traitant du même sujet.

Enfin, les chercheurs ne mentionnent pas de données concernant l'utilisation supplémentaire de sédatifs, la charge de travail pour le personnel infirmier, ou les devenirs défavorables liés à l'agitation au réveil. L'inclusion de ces résultats cliniques aurait permis de mettre en contexte les observations de Yu et coll. En raison de l'absence de ces données, il est difficile de déterminer si le delirium au réveil constitue un problème clinique important suite à l'anesthésie chez l'adulte ou s'il s'agit simplement d'un épiphénomène-ennuyeux certes, mais de courte durée-de l'anesthésie générale.

Malgré ces lacunes, les résultats de Yu soulèvent plusieurs questions dignes d'intérêt. Quel est le mécanisme à l'origine de l'agitation au réveil? La vitesse du réveil de l'anesthésie joue-t-elle un rôle? Un réveil plus rapide après l'anesthésie à l'aide d'un agent inhalé à faible solubilité tel que le sévoflurane est associé à un risque accru d'agitation au réveil chez l'enfant (rapport de cotes [RC] 2,21, IC $95 \%, 1,77-2,77) ;^{11}$ pourtant, l'administration concomitante de midazolam semble n'avoir aucun effet (RC 0,88, IC $95 \%$, 0,44-1,76). ${ }^{12}$ Quelles sont les composantes essentielles de l'analgésie pour éviter l'agitation au réveil? Une méta-analyse récente suggère que les analgésiques actifs au niveau central tels que les opiacés, la kétamine et les agonistes alpha-2 adrénergiques réduisent l'agitation au réveil. ${ }^{12}$ Une technique d'anesthésie régionale, fournissant une analgésie 
sans dépression du système nerveux central, protège-t-elle également le patient de l'agitation au réveil? L'agitation à l'unité de soins post-anesthésiques prédit-elle un delirium plus tard en période postopératoire, ou prédit-elle un DCPO à plus long terme?

S'ils souhaitent aborder ces questions, les chercheurs cliniques doivent avoir recours à des méthodes et des instruments plus rigoureux afin de garantir que leurs mesures ont une validité interne. L'utilisation d'instruments tels que les échelles CAM, PAED et les scores d'agitation en soins critiques donneront des résultats à la fois clairement définis et comparables à d'autres études dans le domaine. En élargissant la portée des recherches au-delà du phénomène en soi afin d'inclure des facteurs tels que l'utilisation des ressources, les événements défavorables et la corrélation à d'autres événements cognitifs postopératoires, les chercheurs seront en mesure de placer l'agitation au réveil dans un contexte clinique adapté. Une fois le problème clinique clairement décrit et son histoire naturelle définie, les travaux sur les mécanismes de l'agitation au réveil pourront progresser. Mille excuses à Shakespeare, mais la recherche cognitive périopératoire ne progressera que s'il y a méthode dans la folie.

Funding Dr. Bryson is supported by the Ottawa Hospital Anesthesia Alternate Funds Association.

Disclosure The author declares no commercial or non-commercial affiliations that are or may be perceived to be a conflict of interest with the work.

\section{References}

1. Inouye SK. Delirium in older persons. N Engl J Med 2006; 354: 1157-65.
2. Steinmetz J, Christensen KB, Lund T, Lohse N, Rasmussen LS, ISPOCD Group. Long-term consequences of postoperative cognitive dysfunction. Anesthesiology 2009; 110: 548-55.

3. Yu $D$, Chai $W$, Sun $X$, Yao L. Emergence agitation in adults: risk factors in 2,000 patients. Can J Anesth 2010; 57. doi:10.1007/ s12630-010-9338-9

4. American Psychiatric Association. Diagnostic and Statistical Manual of Mental Disorders: DSM IV, Fourth Edition, Text Revision. Washington, DC: American Psychiatric Association; 2000 .

5. Inouye SK, van Dyck CH, Alessi CA, Balkin S, Siegal AP, Horwitz $R I$. Clarifying confusion: the confusion assessment method. A new method for detection of delirium. Ann Intern Med 1990; 113: 941-8.

6. Rasmussen LS, Larsen K, Houx P, et al. The International Study of Postoperative Cognitive Dysfunction. The assessment of postoperative cognitive function. Acta Anaesthesiol Scand 2001; 45: 275-89.

7. Lepouse C, Lautner CA, Liu L, Gomis P, Leon A. Emergence delirium in adults in the post-anesthesia care unit. $\mathrm{Br} \mathrm{J}$ Anesth 2006; 96: 747-53.

8. Cohen IT, Hannallah RS, Hummer KA. The incidence of emergence agitation associated with desflurane anesthesia in children is reduced by fentanyl. Anesth Analg 2001; 93: 88-91.

9. Sessler CN, Gosnell MS, Grap MJ, et al. The Richmond Agitation-Sedation Scale: validity and reliability in adult intensive care unit patients. Am J Respir Crit Care Med 2002; 166: 1338-44.

10. Riker RR, Picard JT, Fraser GL. Prospective evaluation of the Sedation-Agitation Scale for adult critically ill patients. Crit Care Med 1999; 27: 1325-9.

11. Kuratani $\mathrm{N}, \mathrm{Oi} Y$. Greater incidence of emergence agitation in children after sevoflurane anesthesia as compared with halothane: a meta-analysis of randomized controlled trials. Anesthesiology 2008; 109: 225-32.

12. Dahmani S, Stany I, Brasher C, et al. Pharmacological prevention of sevoflurane- and desflurane-related emergence agitation in children: a meta-analysis of published studies. Br J Anaesth 2010; 104: 216-23.

13. Sikich N, Lerman J. Development and psychometric evaluation of the pediatric anesthesia emergence delirium scale. Anesthesiology 2004 ; 100: 1138-45. 